\title{
El Ketorolac es el AINE con mayor riesgo de sangrado digestivo
}

Risk of hospitalizacion for upper gastrointestinal tract bleeding associated with ketorolac, other nonsteroidal anti-inflamatory drugs, calcium antagonist, and other antihipertensive drugs.

Archives of Internal Medicine. 1998. 158:33-39.

\section{Objetivo}

Examinar el riesgo de hemorragia digestiva alta y/o perforación asociado con AINES y antihipertensivos en la población general.

\section{Diseño}

Estudio caso control poblacional anidado en un estudio de cohortes con cuatro años de seguimiento.

\section{lugar}

Región de Friuli-Venezia Giulia, Italia.

\section{Participantes}

De los 780.463 residentes permanentes en la región, de 25 a 89 años de edad fueron identificados 1505 pacientes hospitalizados por una hemorragia digestiva alta (HDA). Un grupo control de 20.000 personas fue seleccionado al azar.

\section{Evaluación de factores de riesgo.}

Se excluyeron personas con hospitalización previa por cáncer, coagulopatía, alcoholismo, várices esofágicas, síndrome de Mallory-Weiss, hepatopatía crónica y enteritis regional. Se excluyeron embarazadas durante el período de estudio. El estudio no excluyó pacientes con antecedentes de dispepsia o úlcera.

\section{Medición de resultados principales.}

Los casos de hospitalización por hemorragia digestiva (1505 casos) ó perforación (223 casos), datos demográficos, clínicos y farmacológicos se obtuvieron a través del servicio de datos computarizados de la región.
Resultados principales

Riesgo de HDA según AINE individual

\begin{tabular}{lrrl}
\hline AINE & $n^{\circ}$ Casos & $n^{\circ}$ Controles & $\begin{array}{l}\text { RR ajustado } \\
\text { (IC 95\%) }\end{array}$ \\
Ninguno & 607 & 15242 & 1 \\
\hline Ibuprofeno & 3 & 31 & $2.1(0.6-7.1)$ \\
\hline Diclofenac & 15 & 90 & $2.7(1.5-4.8)$ \\
\hline Naproxeno & 5 & 33 & $4.3(1.6-11.2)$ \\
\hline Tenoxicam & 8 & 35 & $4.3(1.9-9.7)$ \\
\hline Nimesulida & 15 & 90 & $4.4(2.5-7.7)$ \\
\hline Indometacina & 4 & 10 & $5.5(1.6-18.9)$ \\
\hline Piroxicam & 47 & 101 & $9.5(6.5-13.8)$ \\
\hline Ketorolac & 11 & 8 & $24.7(9.6-63.5)$ \\
\hline
\end{tabular}

El ketorolac fue el AINE más asociado a la HDA. El RR fue mayor para la forma IM (28,3 IC95\% 8,7 a 92) que para la oral ( 19,9 IC95\% 4,2 a 93) y para el uso por más de una semana ( 30,3 IC95\% 8,5 a 107,5). El RR de Ketorolac versus todos los otros AINE fue 5,5 ( 2,1 a 14,4).

Dentro de los antihipertensivos, los bloqueantes cálcicos presentan el mayor riesgo de sangrado $(1,7,1,3$ a 2,1) que disminuye a 1,4 cuando se ajusta por marcadores de comorbilidad.

\section{Conclusión}

El excesivo riesgo de complicaciones mayores gastrointestinales asociado con Ketorolac sugiere una desfavorable relación riesgo-beneficio comparado con otros AINE.

Se requieren más datos para definir la relación entre los bloqueantes cálcicos y el pequeño aumento del riesgo de sangrado observado en este estudio.

\section{COMENTARIO}

El ketorolac fue introducido en la década del 90 en nuestro país, y su publicidad se basó en un efecto analgésico más potente que otros AINEs. En un reciente meta-análisis de complicaciones gastrointetinales de los AINE (1), el Ketorolac no fue incluído. Este es el primer estudio poblacional publicado que evalúa el Ketorolac, otros AINE y su asociación con la HDA.

En este estudio El RR de sangrado con el uso de los AINE en general muestra cifras acordes con lo publicado en la literatura (2 -3). La incidencia anual de hospitalización por hemorragia digestiva fue de $1 \mathrm{x}$ 1000 ( cifra similar a la observada por ejemplo en la población general de Canadá ) (4). La incidencia para quienes usaban Ketorolac fue de $20 \times 1000$ personas /año.

El ketorolac presentó mayor riesgo que el Piroxicam y éste fue alto aún a dosis bajas ( menos de $20 \mathrm{mg} /$ día ). Otros estudios publicados mues- tran un leve aumento de sangrado gastrointestinal y por heridas quirúrgicas con Ketorolac EV (5), y alto riesgo de úlcera y erosiones gástricas con respecto a otros AINE (6 -7).

Se aguardan todavía estudios poblacionales en los que se evalúen los efectos de los inhibidores de la Cox-2 y su presumible papel en la menor incidencia de efectos adversos gastrointestinales.

Como siempre, el médico, con el uso juicioso, individualizado y por períodos cortos de AINE tiene la palabra.

Con respecto a los calcio-antagonistas el riesgo de sangrado fue relativamente pequeño $(R R \cong 2$ ) y éste disminuyó a 1.4 cuando fue ajustado por comorbilidad. Aún cuando estos resultados pueden mostrar una tendencia, es necesario contar con evidencia más contundente antes de hacer cualquier recomendación.

Dr. Juan Andrés Torres

Servicio de Clínica Médica. Hospital Italiano de Buenos Aires.

\footnotetext{
Referencias

1. Henry,D. Variability in risk of G-I complications with individual NSAIs drugs. Results of a collaborative meta-analysis. BMJ. 1996, 312:1563-1566.

2. Hawkey, CJ. NSAIs drugs and peptic ulcers: facts and figures multiply, but do they add up?.BMJ. 300:278-284.

3. Bollini,P. The impact of research quality and study design on epidemiologic estimates of the effect of NSAIDs on upper G-I pathology. Arch. Int. Med: $1992,152: 1289-1295$.

4. Pérez Guthan, S. Individual NSAIDs and other risk factors for upper G-I bleeding. Epidemiology. 1997, 8:18-24.

5. Strom, B. L. Parenteral Ketorolac and risk of G-I and operative site bleeding. JAMA. 1996,275: 376-382.

6. Traversa, G. G-I toxicity of different NSAIDs. Epidemiology. 1995, 6:49-54.

7. Lewis, S. Ketorolac in Europe. Lancet. 1994, 343:784.
} 